\title{
Brain Type 1 Cannabinoid Receptor Availability in Patients with Anorexia and Bulimia Nervosa
}

\author{
Nathalie Gérard, Guido Pieters, Karolien Goffin, Guy Bormans, and Koen Van Laere
}

Background: The endocannabinoid system is a possible target in the treatment of eating disorders. We used positron emission tomography to investigate the type 1 cannabinoid receptor (CB1R) in bulimic and anorectic patients.

Methods: We investigated 16 female bulimia nervosa patients $(\mathrm{BN})$ (age $=23.8 \pm 7.1$ years) and 14 female anorexia nervosa patients (AN) (age $=20.5 \pm 3.6$ years) using the selective CB1R ligand $\left[{ }^{18} \mathrm{~F}\right] \mathrm{MK}-9470$. The control group consisted of 19 age-matched women (age $=25.2 \pm$ 8.5 years). Statistical parametric mapping ( $p_{\text {family-wise error }}<.05$ ) and volume-of-interest analyses of CB1R availability were performed.

Results: Global CB1R availability was significantly increased in cortical and subcortical brain areas in AN patients compared with healthy control subjects $(+24.5 \%, p=.0003)$. Regionally, CB1R availability was increased in the insula in both AN and BN patients $(p=.01$ and $p=$ $.0004)$ and the inferior frontal and temporal cortex in AN patients only $(p=.02)$.

Conclusions: Global CB1R upregulation in AN patients is a possible long-term compensatory mechanism to an underactive endocannabinoid system in anorectic conditions. There is a similarity in CB1R dysregulation both in AN and BN in the insular cortex, which is involved in the integration of interoceptive information, gustatory information, reward, and emotion processing.

Key Words: [ $\left.{ }^{18} \mathrm{~F}\right] \mathrm{MK}-9470$, anorexia nervosa, bulimia nervosa, insula, positron emission tomography, type 1 cannabinoid receptor

A norexia nervosa (AN) and bulimia nervosa (BN) are severe psychiatric disorders with prevalence in Western countries of . $7 \%$ and up to $2 \%$, respectively, and a poor clinical outcome. Characteristic symptoms of AN are the refusal to maintain a minimally normal body weight, an intense fear of gaining weight or becoming fat, and a disturbed perception of body shape and size. Bulimia nervosa is characterized by binge-eating episodes and loss of control over eating behavior. In addition, self-esteem in these patients is largely determined by their body shape and weight (1).

Pharmacotherapeutic interventions for $\mathrm{AN}$ and $\mathrm{BN}$ remain unsatisfactory, as there is still a lack of understanding of their pathogenesis. Functional neuroimaging has shown cortical metabolic dysfunction and changes in monoamine neurotransmitter systems (2). While the serotonergic and dopaminergic systems have been studied most intensively, the endocannabinoid neurotransmission system (ECS) has recently been recognized as an important target in both food intake and reward processing (3). The type 1 cannabinoid receptor (CB1R) is abundantly expressed in the central nervous system and induces mainly inhibition of neurotransmission through modulation of presynaptic neurotransmitter release, primarily through retrograde signaling (4). Type 1 cannabinoid receptor inverse agonists inhibit food intake through both central and peripheral mechanisms (5), but their development is halted by undesired central side effects such as increased prevalence of depression and suicidality. In contrast, cannabinoid agonists stimulate food intake in humans and induce beneficial effects in acquired immune deficiency syndrome related anorexia, suggesting altered

From the Division of Nuclear Medicine (NG, KG, KVL), University Hospital and Katholieke Universiteit Leuven, Leuven; University Psychiatric Centre (GP), Katholieke Universiteit Leuven, Eating Disorder Clinic Kortenberg, Kortenberg; and Laboratory for Radiopharmacy (GB), Katholieke Universiteit Leuven, Leuven, Belgium.

Address correspondence to Koen Van Laere, M.D., Ph.D., D.Sc., University Hospitals, Leuven, Division of Nuclear Medicine, Herestraat 49, B-3000 Leuven, Belgium; E-mail: koen.vanlaere@uzleuven.be.

Received Oct 26, 2010; revised Apr 19, 2011; accepted May 9, 2011.
ECS neurotransmission in anorectic conditions (6). Different alleles of the CB1R gene have been associated with restricting and bingeeating/purging AN subtypes (7). Moreover, an increase in plasma levels of the endocannabinoid anandamide has been demonstrated in AN but not in BN (8). Also in animals, an orexigenic effect of CB1R agonists is present, even in the satiated condition (9). In one clinical trial in primary anorexia nervosa, patients received high doses of cannabis' psychoactive component $\Delta^{9}$-tetrahydrocannabinol, but no improvement in food intake or weight was observed (10).

Recently, the CB1R-specific radioligand [ $\left.{ }^{18} \mathrm{~F}\right] \mathrm{MK}-9470$ was developed for CB1R positron emission tomography (PET) imaging and characterized preclinically and clinically (11). Its affinity $(.7 \mathrm{nmol} / \mathrm{L})$ is much higher than the endogenous cannabinoids anandamide and 2-arachidonoylglycerol (2-AG), and its selectivity is several tenfold higher for the CB1R over the type 2 cannabinoid receptor (11). Using this radioligand, we have investigated in vivo CB1R availability in both female AN and BN patients, in comparison with agematched healthy volunteers. Based on current literature on the ECS in eating disorders, we hypothesized that the ECS would be underactive, at least in AN, and a compensatory chronic upregulation of the $C B 1 R$ would be the result.

\section{Methods and Materials}

\section{Participants and Procedure}

Female AN and BN patients were recruited during hospitalization in an inpatient university center for eating disorders. Seven restricting and seven binging-purging $A N$ patients were included, as well as 16 purging BN patients, as diagnosed according to DSM-IV criteria (12). Patients were between 17 and 45 years old. The control group (CON) consisted of 19 healthy age-matched women. Informed consent was obtained from all participants before study investigations, and the study was approved by the local ethics committee and performed according to the World Medical Association Declaration of Helsinki.

All participants were screened for absence of current medical conditions or current psychosis and addiction and were free of any psychoactive medication. Blood and urine testing as performed on the day of PET scanning included screening for benzodiazepines, neuroleptics, opiates (including synthetic), cocaine and metabo- 
lites, amphetamines, and cannabinoids. Magnetic resonance imaging (MRI) was performed to exclude structural brain abnormalities and for voxel-based morphometry to assess possible changes in gray matter concentration (13). Subjects completed the Eating Disorder Evaluation Scale (EDES) and the Eating Disorder Inventory (EDI) to assess psychological and clinical aspects related to eating disorders $(14,15)$.

\section{Radiotracer Characteristics and Preparation}

The radiotracer $\left[{ }^{18} \mathrm{~F}\right]$ MK-9470 (Merck Research Laboratories, West Point, Pennsylvania) is an inverse agonist with a high affinity and specificity for the human CB1R. The precursor for tracer synthesis was obtained from Merck Research Laboratories and subsequent labeling was performed onsite with 2-fluoroethyl bromide as previously described (11). The radioligand had a radiochemical purity of $>95 \%$ and a mean specific radioactivity of $261 \pm 144 \mathrm{GBq} /$ $\mu \mathrm{mol}$.

\section{Imaging Procedure}

All PET acquisitions were performed on an HR+ camera (Siemens, Ehrlangen, Germany). Subjects fasted for at least 4 hours before the PET scan and received on average $306 \pm 55 \mathrm{MBq}$ of $\left[{ }^{18} \mathrm{~F}\right] \mathrm{MK}-9470$ in a slow bolus injection, under standardized injection circumstances. Full dynamic measurements with arterial blood sampling were conducted for the first 10 subjects ( $5 \mathrm{AN}$ and $5 \mathrm{BN}$ ) and for the control subjects, between 0 and 75 minutes and 90 and 120 minutes post injection. These data were used to assess whether a simplified noninvasive quantification procedure (see further), as shown for healthy volunteers, was justified in these patient groups as well (16). Arterial sampling for input curve determination and blood metabolites was done as described previously (16). For the remaining patients, a simplified dynamic acquisition between 90 and 120 minutes post injection was used, without blood sampling. Using a transmission scan $\left({ }^{68} \mathrm{Ge}\right.$ source) for attenuation correction, images with $4 \mathrm{~mm}$ resolution were reconstructed by a standard filtered backprojection algorithm.

\section{Image Processing}

Each subject's dynamic [ $\left.{ }^{18} \mathrm{~F}\right] \mathrm{MK}-9470$ scan was co-registered to the corresponding volumetric magnetic resonance (MR) image. Regional time-activity curves were calculated from a manually defined volume-of-interest (VOI) template to assess kinetic parameters (16). For the subjects with arterial sampling, the fractional uptake value (fractional uptake rate [FUR]), which is proportional to the CB1R total volume of distribution $\left(\mathrm{V}_{\mathrm{T}}\right)$, was calculated as the ratio of total radioactivity concentration in tissue at the end of the scan and the integral of metabolitecorrected plasma radioactivity from time of injection to the end of the scan (16). Also, parametric modified standard uptake value (mSUV) images were generated by summation of the 90 to 120 minutes image data, corrected for injected dose and subject's body weight: $\mathrm{mSUV}=\{$ [activity concentration $(\mathrm{kBq} / \mathrm{mL}) \times($ subject's weight $[\mathrm{kg}]+70) / 2] /$ injected dose $(\mathrm{MBq})\}(17)$. With comparable peripheral tracer metabolization in subjects, this simplified method can be used as indicator of CB1R availability, thereby not requiring invasive blood sampling (16). Figure 1 shows that no difference in FUR versus mSUV relationship was present between both groups, indicating absence of systematic bias or peripheral metabolization effects because of this simplification.

Optimized voxel-based morphometry (VBM) was performed to assess structural gray matter differences between groups (18). Normalized MR images were segmented into gray matter maps and a voxel-

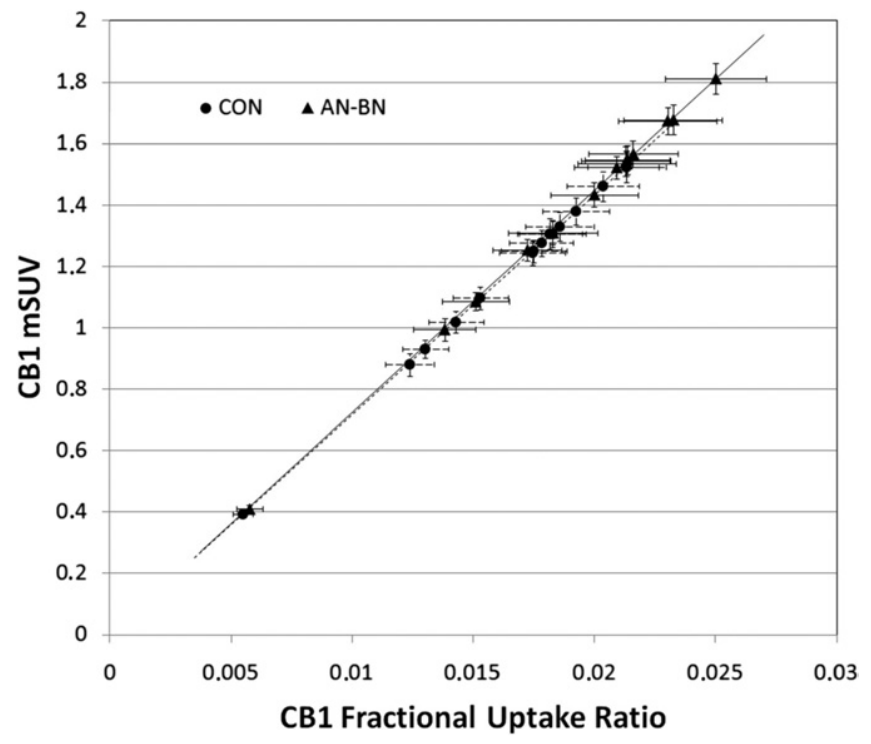

Figure 1. Relationship between simplified quantification using modified standard uptake values and fractional uptake, which is directly proportional to $V_{\mathrm{T}}$ (total receptor distribution volume) (16), for control subjects $(n=19)$ versus a subgroup of patients $(n=10)$. Full lines and triangles $=$ patient data, dotted lines and circles $=$ control subject data. Error bars indicate the standard error of the mean. AN, anorexia nervosa; BN, bulimia nervosa; CB1, type 1 cannabinoid; CON, control group; mSUV, modified standard uptake values.

wise comparison of the local gray matter concentration was performed with Statistical Parametric Mapping version 2 (SPM 2; Wellcome Trust Centre for Neuroimaging, London, United Kingdom).

\section{Data Analysis}

For Statistical Parametric Mapping analysis, spatially normalized gray matter maps and mSUV PET images were smoothed with an isotropic $10 \mathrm{~mm}$ Gaussian kernel and analyzed using a categorical design. Both absolute and regional relative distributions of CB1R availability were investigated. The latter was done by normalizing activity to total cerebral values. For statistical assessment, a relative gray matter analysis threshold of $80 \%$ was set to exclude extracerebral activity, and only significant clusters ( $p_{\text {cluster }}<.05$, corrected for multiple comparisons) were retained, in combination with sufficient localizing power ( $p_{\text {height }}<$ .001 uncorrected for multiple comparisons). The extent threshold $\mathrm{k}_{\text {ext }}$ was set at 50 voxels $\left(.4 \mathrm{~cm}^{3}\right)$ to minimize false-positive small clusters.

Additionally, a VOI analysis was performed by loading a predefined VOI map on all parametric PET images (PMOD; PMOD Inc., Zurich, Switzerland). This VOI map was drawn on an MRI template in Montreal Neurological Institute space, representing cortical Brodmann areas and subcortical gray matter structures (caudate nucleus, putamen, nucleus accumbens, pallidum, thalamus, and hypothalamus). Individual adjustments were performed for the subcortical brain areas based on the individual MR images. Type 1 cannabinoid receptor availability values were compared using analysis of variance and Tukey honestly significant difference post hoc tests $(p<.05)$, by use of Statistica v. 9.0 (StatSoft, Inc., Tulsa, Oklahoma).

With both analysis methods, correlations with EDES and EDI scores were assessed. 
Table 1. Demographic and Clinical Characteristics of Study Participants

\begin{tabular}{|c|c|c|c|}
\hline Parameter & AN & $\mathrm{BN}$ & Control Subjects \\
\hline$n$ & $14 \mathrm{~F}(7 \mathrm{AN}-\mathrm{BP} / 7 \mathrm{AN}-\mathrm{R})$ & $16 \mathrm{~F}(16 \mathrm{BN}-\mathrm{P})$ & $19 \mathrm{~F}$ \\
\hline Age (years) & $20.5 \pm 3.6$ & $23.8 \pm 7.1$ & $25.2 \pm 8.5$ \\
\hline Weight (kg) & $42.5 \pm 6.2^{a, b}$ & $61.2 \pm 10.3^{a}$ & $66.4 \pm 13.1^{b}$ \\
\hline $\mathrm{BMI}\left(\mathrm{kg} / \mathrm{m}^{2}\right)$ & $15.5 \pm 1.3^{a, b}$ & $21.8 \pm 2.5^{a}$ & $23.1 \pm 4.3^{b}$ \\
\hline \multirow[t]{2}{*}{ Menstrual State } & Amenorrhea $(n=14)$ & Normal $(n=14)$ & Normal $(n=19)$ \\
\hline & & Amenorrhea $(n=2)$ & \\
\hline Estimated Disease Duration (years) & $4.2 \pm 4.5$ & $6.3 \pm 6.3$ & - \\
\hline EDES & $n=11$ & $n=13$ & $n=13$ \\
\hline Total & $36.7 \pm 9.5^{b}$ & $37.4 \pm 9.5^{c}$ & $69.5 \pm 6.2^{b, c}$ \\
\hline EDI & $n=13$ & $n=15$ & $n=13$ \\
\hline Total & $87.3 \pm 19.4^{b}$ & $83.4 \pm 30.1^{c}$ & $13.7 \pm 9.6^{b, c}$ \\
\hline
\end{tabular}

Values are given as mean \pm 1 standard deviation. Scores below 55 on the EDES questionnaire indicate pathology; for the EDI, pathology is defined with a cutoff value of 30 or higher. Significant differences $p<.05$.

$\mathrm{AN}$, anorexia nervosa; BMI, body mass index; BN, bulimia nervosa; BP, binging-purging; EDES, Eating Disorder Evaluation Scale; EDI, Eating Disorder Inventory; $F$, female; $P$, purging; $R$, restricting.

${ }^{a} \mathrm{AN}$ versus $\mathrm{BN}$.

${ }^{b} \mathrm{AN}$ versus control subjects.

${ }^{\mathrm{B}} \mathrm{BN}$ versus control subjects.

\section{Results}

\section{Clinical Characteristics}

Demographic and clinical characteristics and EDES/EDI (sub)scores for all subjects are given in Table 1. Weight and body mass index of AN patients were significantly lower compared with the BN patients and healthy control subjects $(p<.001)$. No differences were found in disease duration between both patient groups. Anorexia nervosa and BN patients scored significantly lower on the EDES questionnaire $(p<.001)$ and significantly higher on the EDI questionnaire relative to healthy control subjects $(p<.001)$.

\section{Imaging Results}

Optimized voxel-based morphometry did not detect any significant changes in gray matter concentration between $\mathrm{AN}, \mathrm{BN}$, and CON. Therefore, no partial volume correction was performed on the PET images.

As restricting and binge-purging anorectics showed no significant differences in their demographic characteristics or in CB1R binding, results were analyzed and reported for both subgroups together.

No significant differences in metabolization or metabolite corrected arterial input functions were present between the AN, BN, and CON groups. Figure 1 shows the group comparison of mSUV and FUR values as indicator for the AN, BN, and CON groups. Therefore, the remaining analysis was done using mSUV determinations.
The mean injected [ $\left.{ }^{18} \mathrm{~F}\right] \mathrm{MK}-9470$ dose was $269 \pm 67 \mathrm{MBq}$ for AN, $303 \pm 55 \mathrm{MBq}$ for $\mathrm{BN}$, and $336 \pm 17 \mathrm{MBq}$ for the control group.

Statistical Parametric Mapping analysis showed a whole-brain increase of CB1R availability in the AN group compared with the BN patients and the control group. The average gray matter mSUV of the AN group was increased with $24.5 \%$ compared with control subjects $(p=.0003)$ and with $14.7 \%$ compared with the BN group $(p=.03)$ (Figure 2). The difference between BN and control subjects did not reach significance $(p=.16)$.

Regional analysis after normalizing mSUV to global cerebral uptake showed a relative significant increase in CB1R availability in the bilateral insular cortex of AN and BN patients in comparison with healthy control subjects (Figure 3). Only in AN, clustered CB1R increases were present in the inferior frontal cortex (Brodmann area 45) and in the inferior temporal cortex (Brodmann area 20) compared with the control volunteers. Cluster location, significance, and group relative differences are given in Table 2. No significant differences were found between both patient groups.

\section{Correlation Analyses}

Regional relative CB1R availability was positively correlated with the EDI subscale drive for thinness in the superior temporal brain area of AN patients $\left(r=.86, p_{\text {height }}<.001\right.$ ) (Figure 4$)$, but no significant correlations were present for $\mathrm{BN}$ patients.

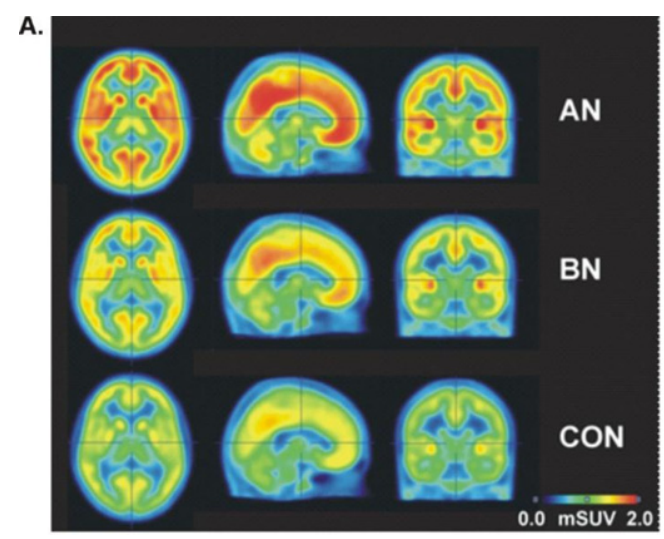

B.

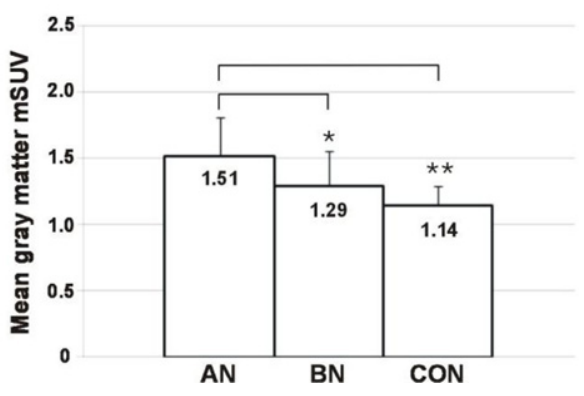

Figure 2. (A) Mean parametric images of global $\left[{ }^{18} \mathrm{~F}\right] \mathrm{MK}-9470$ binding in the anorexia nervosa (AN), bulimia nervosa (BN), and control group (CON). Modified standard uptake values (mSUV) are indicated by the color bar. (B) Quantitative representation of mean gray matter mSUV (+ 1 SD) for each group. ${ }^{* * A N}>$ CON, $p<.001$; ${ }^{*} \mathrm{AN}>\mathrm{BN}, p<$ .05. AN: $n=14, \mathrm{BN}: n=16$, control subjects: $n=19$. 
A.
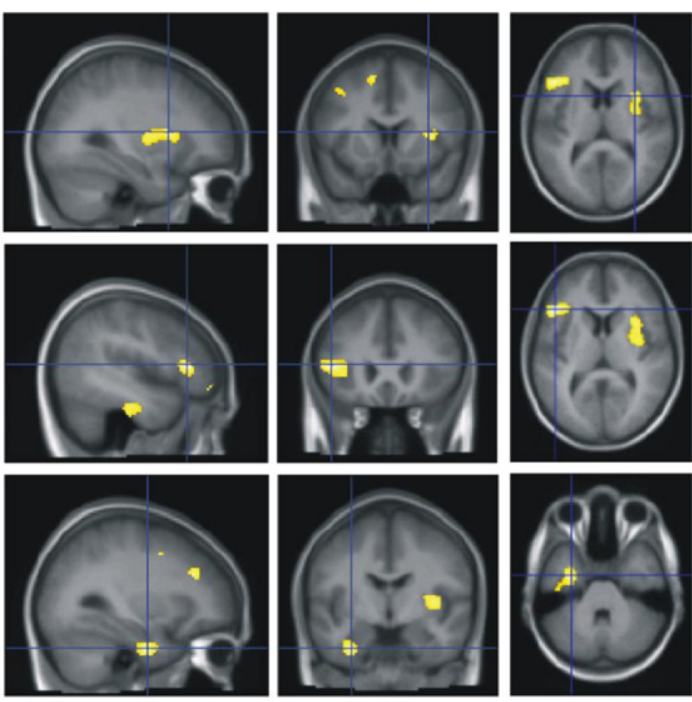

B.
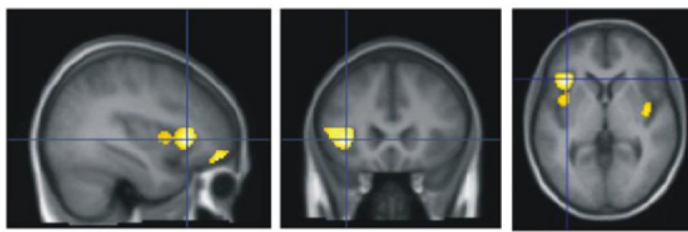

L

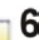

Figure 3. (A) Increases in relative type 1 cannabinoid receptor availability in the insular, inferofrontal, and inferotemporal pole of anorexia nervosa (AN) patients and (B) In the insular cortex of bulimia nervosa (BN) patients compared with healthy control subjects. Statistical Parametric Mapping T-maps are overlaid on a T1 magnetic resonance imaging template at $p_{\text {height }}<.001$ (uncorrected); T values are indicated by the color bar. Cluster extent threshold is 50 voxels. AN: $n=14, \mathrm{BN}: n=16$, control subjects: $n=19$. L, left; $\mathrm{R}$, right.

\section{Discussion}

In this study, we observed strong changes in cerebral CB1R availability in vivo in female anorectic and bulimic patients in comparison with age-matched healthy volunteers. A widespread involvement of the ECS in anorexia nervosa is not surprising when considering its role in feeding modulation, in hedonics of food intake, and generally in reward (19). Stimulation of endocannabinoid signaling has an orexigenic effect, but the ECS is also physio- logically involved in energy homeostasis through food intake (3). Previously, it has been hypothesized that the ECS is hypoactive in anorectic conditions $(20,21)$, based on several indirect arguments. Cannabinoid agonists are used as appetite stimulants in cancer and AIDS patients, likely compensating for endocannabinoid hypoactivity during anorectic conditions that accompany these diseases (6). Type 1 cannabinoid receptor inactivation by blockade or genetic deletion is a strategy used to suppress eating by decreasing hedonic aspects of food intake, suggesting the importance of tonic endocannabinoid signaling for normal feeding (9). Kirkham et al. (22), addressing short-term effects of starvation, reported an increase of hypothalamic 2-AG concentration after 24 hours in the rat brain. Hanus et al. (23) found a whole brain decrease of 2-AG following 12 days of food restriction in mice, including hypothalamic and hippocampal regions. This could reflect adaptive strategies to cope with short or long-lasting food deprivation. In the short term, elevated levels of 2-AG may be beneficial to trigger searching for food and eating behavior, while in the context of a prolonged starvation, survival may be aided by downregulating this orexigenic signal and reducing appetite and motivation to eat (24). Finally, at the opposite side of the eating disorder spectrum in conditions of hyperphagia and obesity, arguments for a hyperactive ECS and CB1R overstimulation have been put forward $(3,25)$. The presumed ECS hypoactivity could be accompanied by a chronic upregulation of CB1R expression, as is also seen in other $G$ protein-coupled receptor systems (e.g., upregulation of striatal D2 receptors in Parkinson's disease). Albeit that decreased endocannabinoid content could also drive a decrease in CB1R expression or that the change in CB1R expression may occur independently from changes in the endocannabinoid content (26), a compensatory receptor upregulation is plausible from a large intracellular CB1R reserve (27).

At odds with this hypothesis, in AN patients, elevated plasma levels of anandamide were found (8), but it is uncertain whether peripheral levels reflect the central nervous system status. Furthermore, little success was obtained in a single $\Delta^{9}$-tetrahydrocannabinol trial in anorectics, but the dosage used in this study may have been too high and therefore even have anorexigenic properties $(10,28)$.

Possible hormonal effects on the global ECS activity in AN patients should also be considered. Ghrelin is increased in AN, while leptin is decreased in $A N$ but not in $B N(8,29,30)$. In rodents, a downregulating effect on the CB1R through leptin signaling has been described (31), while in animal models on obesity, defective leptin signaling has been associated with regionally increased CB1R

Table 2. Cluster $p$ Values and Peak Locations for Statistical Parametric Mapping Comparisons of Regional CB1R Binding Between Patient Groups and Volunteers

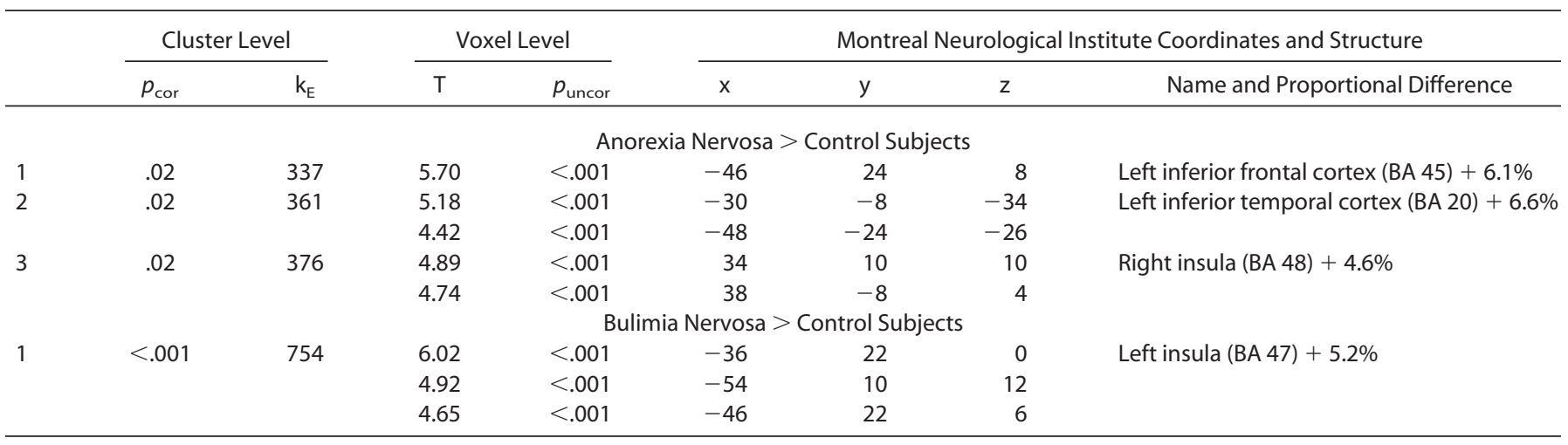

$\mathrm{AN}, n=14 ; \mathrm{BN}, n=16 ;$ volunteers, $n=19$.

$\mathrm{BA}$, Brodmann area; CB1R, type 1 cannabinoid receptor; $\mathrm{k}_{\mathrm{E}}$, cluster extent; $p_{\text {cor, }}$ corrected $p$ value; $p_{\text {uncor' }}$ uncorrected $p$ value. 


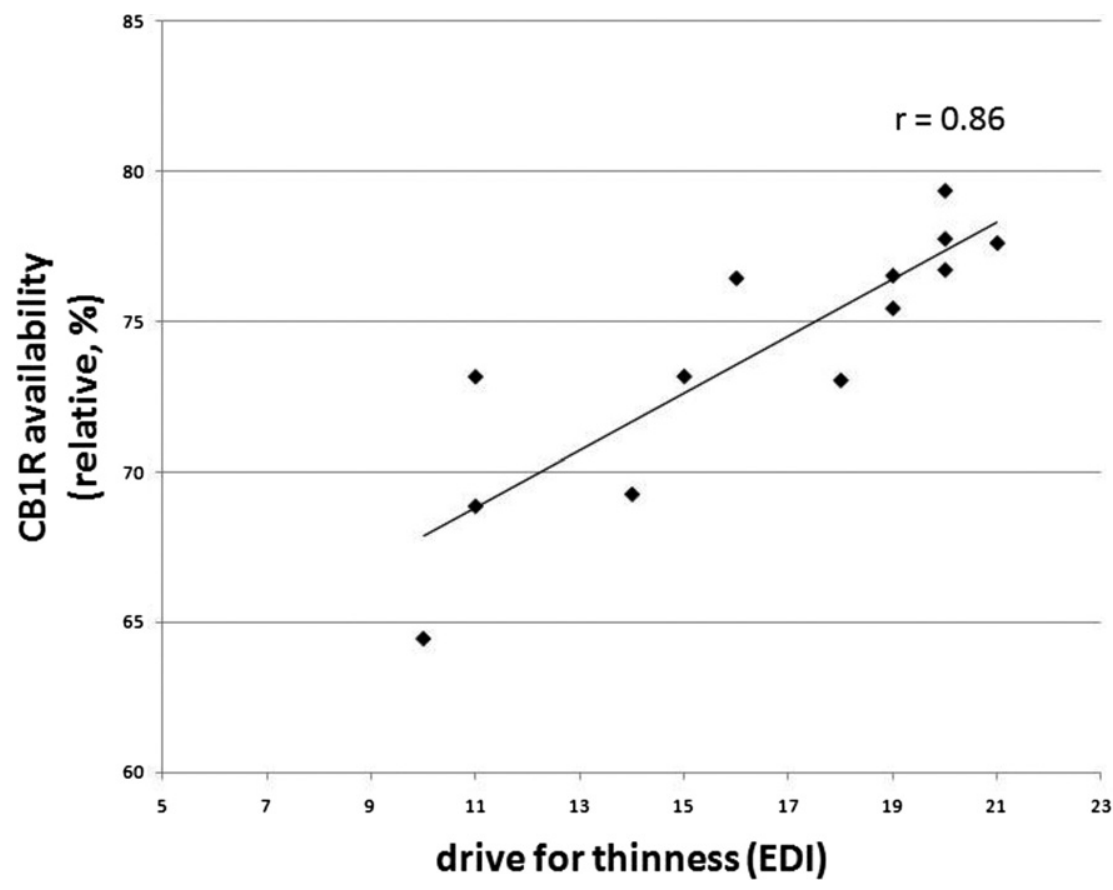

Figure 4. Positive correlation of normalized modified standard uptake values with the Eating Disorder Inventory (EDI) subscale drive for thinness in the right superior temporal cortex of anorexia nervosa (AN) patients (BA 22, $r=.86$ ). AN: $n=14$. BA, Brodmann area; CB1R, type 1 cannabinoid receptor.

binding (32,33). Moreover, higher anandamide turnover may contribute to a compensatory CB1R upregulation if fatty acid amide hydrolase (responsible for the inactivation of anandamide) is not subjected to an inhibitory influence of leptin, as seen in human $T$ lymphocytes (34), or of estrogen. Low estrogen levels are seen in AN patients in the amenorrheic state (35). In contrast, in our population of BN patients, only two presented with amenorrhea.

Regarding regional changes in CB1R availability, we found an insular increase in both groups. The insula is a key area in the neural control of interoception, i.e., the sense of the body's physiological condition, reward, and emotion processing, and its anterior part encompasses the primary taste cortex. Consistent with this knowledge, the insula has been implicated in neural responses to hunger, in the sensory processing of food intake (taste, smell, texture), and in modulation of the rewarding value of food (36). Other symptoms commonly found in AN and BN patients, such as a distorted body perception, lack of insight, and denial of illness or elevated pain thresholds, could also be manifestations of abnormal interoceptive awareness and thus insular dysfunction (37). Insular involvement in eating disorders has been described in functional magnetic resonance imaging studies using symptom provocation strategies with pictures of food, high-calorie drinks, or the subject's own distorted body image $(38-40)$. Our findings further suggest a role for insular cannabinoid signaling dysfunctions in the altered relationship toward food and its rewarding value, which is characteristic for AN and BN patients.

Whether eating disorder pathology, rather than comorbid disturbances in mood and anxiety, can be causally linked to the observed insular dysfunction in our patients remains unclear. The insular cortex has been implicated in obsessive-compulsive disorder (OCD) and more generally in anxiety but also in depression (36). Evaluation of the CB1R in noneating-disordered patients with depression or OCD should further elucidate this issue. Some studies reported direct cannabinoid-related measures in mood and anxiety disorders. Prefrontal CB1R brain density was increased in depressed suicide victims (41) and preclinically in rodent models exposed to chronic stress (26). As endocannabinoid deficiency is suggested to be involved in depressive syndromes (28) and cannabinoids might reduce OCD symptoms (42), this may indicate potential cannabinoid-related disturbances both in those disorders and in eating disorders.

Besides this common main finding in the AN and BN patients, regional differences also were found, i.e., increased CB1R availability in the inferior frontal and inferior temporal cortex of AN patients. In $\mathrm{AN}$, and to a much lesser extent in $\mathrm{BN}$, dysfunction in frontal and temporal areas related to executive functioning and emotional processing has been observed for several other in vivo neurochemical markers, e.g., local hypoperfusion, decreased glucose metabolism, and decreased serotonin-2A neuroreceptor binding potential (for review, see [2]). Neuropsychological deficits present in AN patients, such as problem-solving or attention disabilities, are often related to changes in the frontal cortex because of its mediating role herein (43). This altered cognitive processing could also influence the responsiveness to emotional stimuli mediated in (medial) temporal areas, for example, the decreased experience in food pleasantness as observed in AN patients. In parallel, recently increased brain histamine $\mathrm{H} 1$ receptor binding has been reported in AN patients in limbic brain and in the prefrontal, orbitofrontal, and temporal cortex (44). Our findings also corroborate a recent study describing increased serotonin-1A receptor binding in parts of the frontal and temporal cortices of lean AN patients and suggesting frontotemporal impairment to be involved in the pathophysiology of AN (45). Moreover, we found a positive correlation of CB1R binding in the superior temporal brain area of the AN patients with the scores on the drive for thinness EDI subscale, which represents a core psychopathological feature of the disease. Anorexia nervosa and BN patients could present differences in their motivation for dieting in the fact that rather than the drive to be thinner as in $\mathrm{AN}$, it is the drive to be objectively thin that animates BN patients (46). Anorexia nervosa patients could be motivated more by a fear of gaining weight 
compared with BN patients, meaning a different emotional involvement in this process.

Some limitations of this study have to be considered. Regarding completeness of behavioral variables, not all patients fully completed their questionnaires (as detailed in Table 1). However, this small group of missing data are unlikely to have impacted on the results, as $A N$ and $B N$ patients are well known to differ on these EDES and EDI measurements.

Regarding the state of hunger of the participants, the eating disordered patients were not deprived from food for a longer time than the control subjects, as they were investigated at the moment they were hospitalized with a normalized meal pattern.

Also, a number of potential confounds regarding radiotracer quantification are present. The large differences in body weight between patient groups and control subjects may represent differences in distribution volume of the tracer, e.g., in fatty tissues. There was no difference in metabolized fraction over time for either group. Furthermore, metabolite-corrected plasma input curves were calculated for a subgroup of patients. The area under these input curves was, on average, $417.6 \pm 133.2$ for $A N, 291.9 \pm 117.5$ for $\mathrm{BN}$, and $378.0 \pm 99.8$ for control subjects. Analysis of variance did not detect significant differences in plasma area under the curve between groups. In Figure 5, we also quantified CB1R availability with the FUR, to address these potential confounds, and observed similar results. However, the FUR differences were not significant, most probably because of the limited size of this subset and the slightly higher interindividual variance in FUR measures compared with mSUV (see also [16]). With the absence of tracer delivery or metabolization effects, we, however, demonstrated the reliability of mSUV derived values. Tracer dosing has been accounted for in both mSUV and FUR quantification by injected activity normalization.
We found no differences in brain volume in the patient groups. Many earlier structural neuroimaging studies have reported significant differences in brain volumes between $\mathrm{AN}, \mathrm{BN}$, and control subjects, but these were methodologically constrained and it was not always clear whether these changes were global, regional, or white or gray matter related (47). Since the introduction of automated VBM for morphometric MRI studies, several recent studies have been conducted in nonrecovered eating disorder patients. However, these studies show lack of consistency and results of VBM analyses in ill eating disordered patients must be interpreted cautiously. For example, one study in AN patients reported a gray matter reduction only in the extrastriate body area and another study reported gray matter changes at several temporal and parietal locations, while no structural abnormalities were found in $\mathrm{BN}$ $(48-50)$. We also used VBM but failed to find significant structural brain differences in our AN and BN patient groups. Potential confounders, aside from group size, may have been differences in age, disease duration, and diagnostic severity.

Concerning the correlation analyses, multiple testing correction was done per correlation using standard $p$ cluster values $<.05$ corrected for multiple comparisons. Only results with a significant $p$ cluster value were retained, as is commonly done in PET/functional magnetic resonance imaging studies. An additional Bonferroni correction was not applied because of its high severity for investigation of plausible correlations in an explorative way.

Finally, causality of the observed effects in this cross-sectional study cannot be assessed and a follow-up study in AN and BN patients to investigate state or trait characteristics of the observed changes in CB1R availability is warranted. In line with our interpretation of increased CB1R availability as a compensatory mechanism to counteract ECS hypoactivity, we hypothesize that these changes may be reversible and illness-dependent. Our own animal microPET

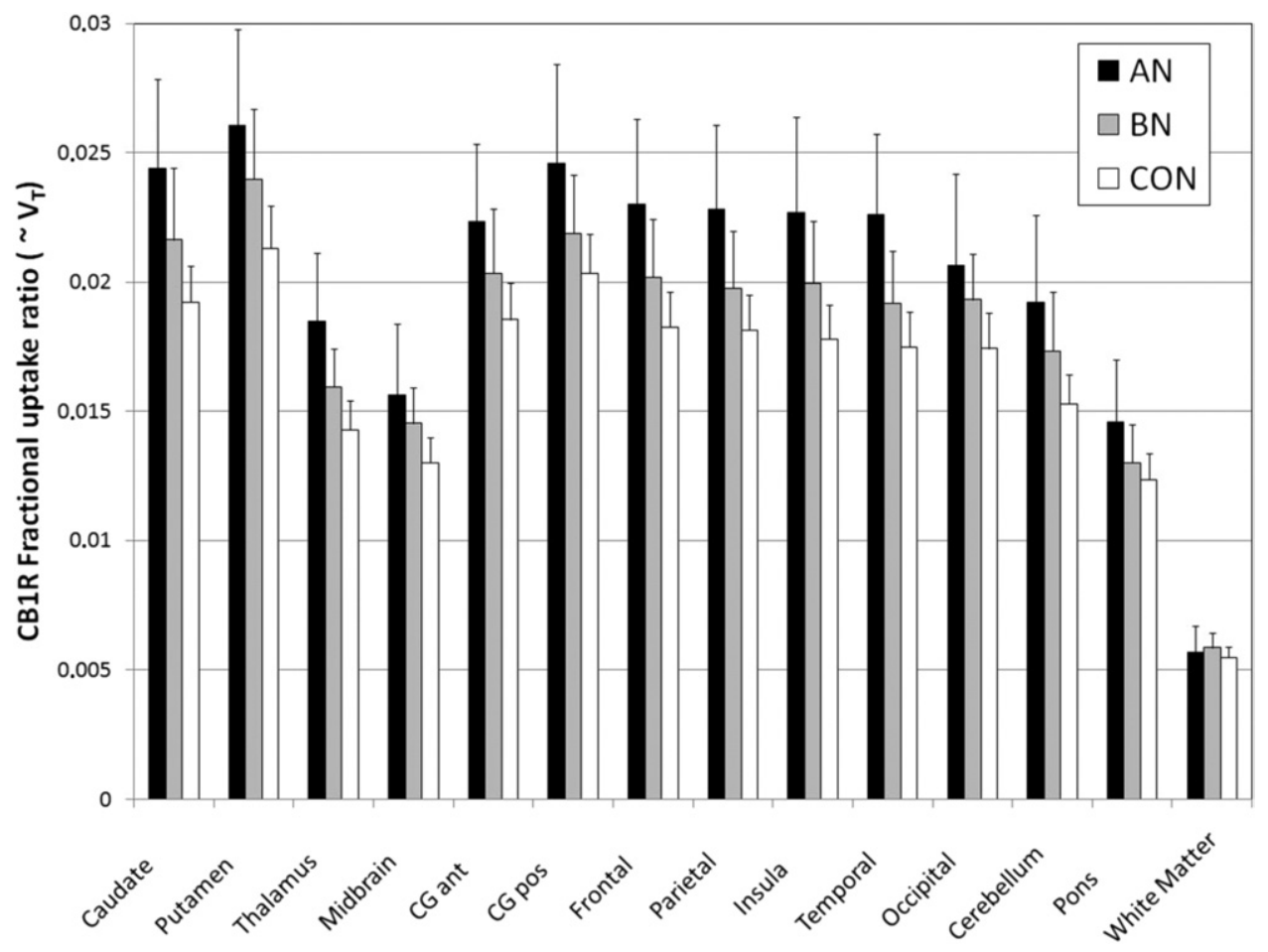

Figure 5. Bar chart of type 1 cannabinoid receptor fractional uptake ratio per volume-of-interest for a smaller subset of anorexia nervosa (AN) and bulimia nervosa (BN) patients and for control subjects (CON). Fractional uptake ratio is increased but not significantly different in all cerebral regions of AN patients compared with the other groups, except in the white matter. AN: $n=5, \mathrm{BN}: n=6, \mathrm{CON}: n=19$. CB1R, type 1 cannabinoid receptor; CG ant, anterior cingulate gyrus; $C G$ pos, posterior cingulate gyrus; $\mathrm{V}_{\mathrm{T}}$, total receptor distribution volume. 
studies in an activity-based anorexia rat model showed reversibility of increased CB1R availability upon recovery from the anorectic state (N.G. et al., unpublished data; June 2010).

New studies with related drug compounds, such as CB1R agonists or fatty acid amide hydrolase or monoacylglycerol lipase inhibitors, and accounting for dose-dependent cannabinoid effects and reduced psychotropic side effects may lead to beneficial therapeutic approaches for eating disorders. As anorexia nervosa does not imply a loss of appetite, beneficial effects of altering cannabinoid signaling might be more related to restoring homeostatic food intake or positively mediating rewarding aspects of food intake. To support this further, in vivo direct or indirect information on the concentration of endocannabinoid ligands in the central nervous system of control subjects and eating disordered patients is warranted.

This work was supported by the Research Council of the Katholieke Universiteit Leuven (OT/05/58). KG is Research Assistant and KVL is Senior Clinical Investigator of the Flemish Fund of Scientific Research.

We are grateful to all study participants. We thank the personnel of the Centre for Eating Disorders and the positron emission tomography radiopharmacy and nuclear medicine team of the University Hospitals Leuven for their skilled collaboration. Merck Research Laboratories is acknowledged for the availability of the $\left[{ }^{18} \mathrm{~F}\right] \mathrm{MK}-9470$ precursor and nonradioactive standard.

$K V L$ received research grants from Merck, Inc., GE Healthcare, and Johnson \& Johnson. GB received research grants from Merck, Inc. and Johnson \& Johnson. All other authors report no biomedical financial interests or potential conflicts of interest.

1. Fairburn CG, Harrison PJ (2003): Eating disorders. Lancet 361:407-416.

2. van Kuyck K, Gérard N, Van Laere K, Casteels C, Pieters G, Gabriëls L, Nuttin B (2009): Towards a neurocircuitry in anorexia nervosa: Evidence from functional neuroimaging studies. J Psychiatr Res 43:1133-1145.

3. Di Marzo V, Ligresti A, Cristino L (2009): The endocannabinoid system as a link between homoeostatic and hedonic pathways involved in energy balance regulation. Int J Obes (Lond) 33(suppl 2): S18--S24.

4. Wilson RI, Nicoll RA (2002): Endocannabinoid signaling in the brain. Science 296:678-682.

5. Addy C, Wright H, Van Laere K, Gantz I, Erondu N, Musser BJ, et al. (2008): The acyclic CB1R inverse agonist taranabant mediates weight loss by increasing energy expenditure and decreasing caloric intake. Cell Metab 7:68-78.

6. Beal JE, Olson R, Lefkowitz L, Laubenstein L, Bellman P, Yangco B, et al. (1997): Long-term efficacy and safety of dronabinol for acquired immunodeficiency syndrome-associated anorexia. J Pain Symptom Manage 14:7-14.

7. Siegfried Z, Kanyas K, Latzer Y, Karni O, Bloch M, Lerer B, Berry EM (2004): Association study of cannabinoid receptor gene (CNR1) alleles and anorexia nervosa: Differences between restricting and binging/purging subtypes. Am J Med Genet B Neuropsychiatr Genet 125B:126-130.

8. Monteleone P, Matias I, Martiadis V, De Petrocellis L, Maj M, Di Marzo V (2005): Blood levels of the endocannabinoid anandamide are increased in anorexia nervosa and in binge-eating disorder, but not in bulimia nervosa. Neuropsychopharmacology 30:1216-1221.

9. Horcajadas FA (2007): Cannabinoids in eating disorders and obesity. Mol Neurobiol 36:113-128.

10. Gross H, Ebert MH, Faden VB, Goldberg SC, Kaye WH, Caine ED, et al. (1983): A double-blind trial of delta 9-tetrahydrocannabinol in primary anorexia nervosa. J Clin Psychopharmacol 3:165-171.

11. Burns HD, Van Laere K, Sanabria-Bohórquez S, Hamill TG, Bormans G, Eng WS, et al. (2007): [18F]MK-9470, a positron emission tomography (PET) tracer for in vivo human PET brain imaging of the cannabinoid-1 receptor. Proc Natl Acad Sci U S A 104:9800-9805.

12. American Psychiatric Association(2000): Diagnostic and Statistical Manual of Mental Disorders DSM-IV-TR (text Revision), 4th ed. Washington, DC: American Psychiatric Publishing.
13. Wagner A, Greer P, Bailer UF, Frank GK, Henry SE, Putnam K, et al. (2006): Normal brain tissue volumes after long-term recovery in anorexia and bulimia nervosa. Biol Psychiatry 59:291-293.

14. Vandereycken W (1993): The Eating Disorder Evaluation Scale (EDES). Eat Disord 1:115-122.

15. Garner DM (2007): Development and validation of a multidimensional Eating Disorder Inventory for anorexia nervosa and bulimia. Int J Eat Disord 2:15-34.

16. Sanabria-Bohorquez SM, Hamill TG, Goffin K, De Lepeleire I, Bormans G, Burns HD, Van Laere K (2010): Kinetic analysis of the cannabinoid-1 receptor PET tracer [(18)F]MK-9470 in human brain. Eur J Nucl Med Mol Imaging 37: 920-933.

17. Thie JA, Hubner KF, Isidoro FP, Smith GT (2007): A weight index for the standardized uptake value in 2-deoxy-2-[F-18]fluoro-D-glucose-positron emission tomography. Mol Imaging Biol 9:91-98.

18. Ashburner J, Friston KJ (2000): Voxel-based morphometry-the methods. Neuroimage 11:805-821.

19. Solinas M, Goldberg SR, Piomelli D (2008): The endocannabinoid system in brain reward processes. Br J Pharmacol 154:369-383.

20. Di Marzo V (2008): Targeting the endocannabinoid system: To enhance or reduce? Nat Rev Drug Discov 7:438-455.

21. van der Stelt M, Di Marzo V (2003): The endocannabinoid system in the basal ganglia and in the mesolimbic reward system: Implications for neurological and psychiatric disorders. Eur J Pharmacol 480:133-150.

22. Kirkham TC, Williams CM, Fezza F, Di Marzo V (2002): Endocannabinoid levels in rat limbic forebrain and hypothalamus in relation to fasting, feeding and satiation: Stimulation of eating by 2-arachidonoyl glycerol. Br J Pharmacol 136:550-557.

23. Hanus L, Avraham Y, Ben-Shushan D, Zolotarev O, Berry EM, Mechoulam $R$ (2003): Short-term fasting and prolonged semistarvation have opposite effects on 2-AG levels in mouse brain. Brain Res 983:144-151.

24. Fride E, Bregman T, Kirkham TC (2005): Endocannabinoids and food intake: Newborn suckling and appetite regulation in adulthood. Exp Biol Med (Maywood) 230:225-234.

25. Pagotto U, Vicennati V, Pasquali R (2005): The endocannabinoid system and the treatment of obesity. Ann Med 37:270-275.

26. Hill MN, Patel S, Carrier EJ, Rademacher DJ, Ormerod BK, Hillard CJ, Gorzalka BB (2005): Downregulation of endocannabinoid signaling in the hippocampus following chronic unpredictable stress. Neuropsychopharmacology 30:508-515.

27. Gifford AN, Bruneus M, Gatley SJ, Lan R, Makriyannis A, Volkow ND (1999): Large receptor reserve for cannabinoid actions in the central nervous system. J Pharmacol Exp Ther 288:478 - 483.

28. Hill MN, Hillard CJ, Bambico FR, Patel S, Gorzalka BB, Gobbi G (2009): The therapeutic potential of the endocannabinoid system for the development of a novel class of antidepressants. Trends Pharmacol Sci 30:484493.

29. Monteleone P, Serritella C, Martiadis V, Scognamiglio P, Maj M (2008): Plasma obestatin, ghrelin, and ghrelin/obestatin ratio are increased in underweight patients with anorexia nervosa but not in symptomatic patients with bulimia nervosa. J Clin Endocrinol Metab 93:4418-4421.

30. Kola B, Farkas I, Christ-Crain M, Wittmann G, Lolli F, Amin F, et al. (2008): The orexigenic effect of ghrelin is mediated through central activation of the endogenous cannabinoid system. PLOS ONE 3:e1797.

31. Jelsing J, Larsen PJ, Vrang N (2009): The effect of leptin receptor deficiency and fasting on cannabinoid receptor 1 mRNA expression in the rat hypothalamus, brainstem and nodose ganglion. Neurosci Lett 463: 125-129.

32. Di Marzo V, Goparaju SK, Wang L, Liu J, Bátkai S, Járai Z, et al. (2001): Leptin-regulated endocannabinoids are involved in maintaining food intake. Nature 410:822-825.

33. Thanos PK, Ramalhete RC, Michaelides M, Piyis YK, Wang GJ, Volkow ND (2008): Leptin receptor deficiency is associated with upregulation of cannabinoid 1 receptors in limbic brain regions. Synapse 62:637-642.

34. Maccarrone M, Di Rienzo M, Finazzi-Agrò A, Rossi A (2003): Leptin activates the anandamide hydrolase promoter in human $\mathrm{T}$ lymphocytes through STAT3. J Biol Chem 278:13318-13324.

35. Golden NH, Carlson JL (2008): The pathophysiology of amenorrhea in the adolescent. Ann N Y Acad Sci 1135:163-178.

36. Nagai M, Kishi K, Kato S (2007): Insular cortex and neuropsychiatric disorders: A review of recent literature. Eur Psychiatry 22:387-394.

37. Kaye W (2008): Neurobiology of anorexia and bulimia nervosa. Physiol Behav 94:121-135. 
38. Ellison Z, Foong J, Howard R, Bullmore E, Williams S, Treasure J (1998): Functional anatomy of calorie fear in anorexia nervosa. Lancet 352:1192.

39. Sachdev P, Mondraty N, Wen W, Gulliford K (2008): Brains of anorexia nervosa patients process self-images differently from non-self-images: An fMRI study. Neuropsychologia 46:2161-2168.

40. Redgrave GW, Bakker A, Bello NT, Caffo BS, Coughlin JW, Guarda AS, et al (2008): Differential brain activation in anorexia nervosa to Fat and Thin words during a Stroop task. Neuroreport 19:1181-1185.

41. Hungund BL, Vinod KY, Kassir SA, Basavarajappa BS, Yalamanchili $R$, Cooper TB, et al. (2004): Upregulation of CB1 receptors and agoniststimulated [35S]GTPgammaS binding in the prefrontal cortex of depressed suicide victims. Mol Psychiatry 9:184-190.

42. Schindler F, Anghelescu I, Regen F, Jockers-Scherubl M (2008): Improvement in refractory obsessive compulsive disorder with dronabinol. Am J Psychiatry 165:536-537.

43. Lena SM, Fiocco AJ, Leyenaar JK (2004): The role of cognitive deficits in the development of eating disorders. Neuropsychol Rev 14:99-113.

44. Yoshizawa M, Tashiro M, Fukudo S, Yanai K, Utsumi A, Kano M, et al. (2009): Increased brain histamine $\mathrm{H} 1$ receptor binding in patients with anorexia nervosa. Biol Psychiatry 65:329-335.
45. Galusca B, Costes N, Zito NG, Peyron R, Bossu C, Lang F, et al. (2008): Organic background of restrictive-type anorexia nervosa suggested by increased serotonin (1A) receptor binding in right frontotemporal cortex of both lean and recovered patients: [(18)F]MPPF PET scan study. Biol Psychiatry 64:1009-1013.

46. Chernyak Y, Lowe MR (2010): Motivations for dieting: Drive for thinness is different from drive for objective thinness. J Abnorm Psychol 119:276 281.

47. Stamatakis EA, Hetherington MM (2003): Neuroimaging in eating disorders. Nutr Neurosci 6:325-334.

48. Castro-Fornieles J, Bargalló N, Lázaro L, Andrés S, Falcon C, Plana MT, Junqué C (2009): A cross-sectional and follow-up voxel-based morphometric MRI study in adolescent anorexia nervosa.J Psychiatr Res 43:331340.

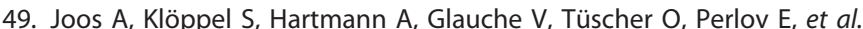
(2010): Voxel-based morphometry in eating disorders: Correlation of psychopathology with grey matter volume. Psychiatry Res 182:146-151.

50. Suchan B, Busch M, Schulte D, Grönemeyer D, Herpertz S, Vocks S (2010): Reduction of gray matter density in the extrastriate body area in women with anorexia nervosa. Behav Brain Res 206:63-67. 